\title{
Isolation of cholesterol-dependent, multidrug-resistant Candida glabrata strains from blood cultures of a candidemia patient in Kuwait
}

\author{
Ziauddin Khan ${ }^{1 *}$, Suhail Ahmad', Leena Joseph ${ }^{1}$ and Khaled Al-Obaid ${ }^{2}$
}

\begin{abstract}
Background: Candida glabrata has emerged as an important human pathogen associated with systemic and mucosal infections. Here, we describe isolation of two cholesterol-dependent Candida glabrata strains from a candidemia patient which failed to grow on the media devoid of a cholesterol source.

Methods: Both the isolates were recovered from BACTEC Plus Aerobic/F blood culture bottles of a candidemic patient. Since these isolates failed to grow on Sabouraud dextrose agar, Mueller-Hinton agar and RPMI 1640 agar media, their definitive identification required PCR sequencing of the internally transcribed spacer (ITS) 1 and ITS2 regions of rDNA and the D1/D2 region sequences within $26 \mathrm{~S}$ rRNA gene. The cholesterol auxotrophy was determined by their ability to grow on media containing a cholesterol source. The minimum inhibitory concentrations (MICs) to antifungal agents were determined by Etest.

Results: The identity of the isolates was confirmed by sequencing of the ITS1 and ITS2 regions of rDNA and the D1/D2 region sequences within $26 \mathrm{~S}$ rRNA gene and also by matrix-assisted laser desorption and ionization-timeof-flight mass spectrometry with $99.9 \%$ confidence value. Both the isolates showed good growth only when media were supplemented with cholesterol, oxbile or blood. Additionally, these isolates were resistant to amphotericin B (MIC $\geq 32 \mu \mathrm{g} / \mathrm{ml}$ ), fluconazole (MIC $\geq 256 \mu \mathrm{g} / \mathrm{ml}$ ), voriconazole (MIC $\geq 32 \mu \mathrm{g} / \mathrm{ml})$, itraconazole (MIC $\geq 32 \mu \mathrm{g} / \mathrm{ml}$ ), and posaconazole (MIC $\geq 32 \mu \mathrm{g} / \mathrm{ml}$ ), but susceptible to caspofungin (MIC range 0.064 to $0.19 \mu \mathrm{g} / \mathrm{ml}$ ).

Conclusion: This appears to be the first report on isolation of cholesterol-dependent strains of $C$. glabrata from a candidemia patient exhibiting resistance to azoles and amphotericin B. Further, the report demonstrates that induction of cholesterol/sterol auxotrophy is associated with resistance to antifungal drugs targeting ergosterol biosynthesis. These observations may have therapeutic implications for the treatment of infections caused by such C. glabrata strains.
\end{abstract}

Keywords: Candida glabrata, Drug resistance, Cholesterol auxotrophy, Candidemia

\section{Background}

Candida glabrata has emerged as the second most important yeast associated with mucosal and systemic infections in critically ill patients in some tertiary care hospitals in North America [1]. The species is intrinsically less susceptible to azoles and it is generally believed that extensive topical and systemic use of these drugs might have contributed to its rising incidence as a human pathogen.

\footnotetext{
* Correspondence: zkhan@hsc.edu.kw

'Department of Microbiology, Faculty of Medicine, Kuwait University, P. O. Box 24923, Safat 13110, Kuwait

Full list of author information is available at the end of the article
}

There are multiple mechanisms that impart resistance against azoles. These include alterations in ERG11 gene that encodes for 14- $\alpha$-methyl sterol demethylase in ergosterol biosynthesis and/or upregulation of efflux pumps encoded by $C g C D R 1$ and $C g C D R 2$ genes [2-4]. Additionally, alterations in $E R G 3$ gene encoding $\Delta^{5,6}$ sterol desaturase have been noted in strains carrying mutations in ERG11 to allow survival under aerobic conditions [5]. Here, we describe the isolation of two C. glabrata strains from bloodstream of a candidemia patient which required exogenous cholesterol/sterol for growth in media that are routinely used in clinical mycology laboratories. 


\section{Methods}

\section{Isolation of C. glabrata strains}

The isolates, Kw1018/12 and Kw1154/12, were obtained 9 days apart from two blood samples at Al-Amiri hospital, Kuwait. Initially, the isolates grew slowly in BACTEC Plus blood culture bottles with detection time of 54 and 75 hours, respectively and were tentatively identified by Vitek2 yeast identification system as C. glabrata. The isolates were received at Mycology Reference Laboratory, Faculty of Medicine, Kuwait University for further identification and antifungal susceptibility testing. When the isolates were subcultured on Sabouraud dextrose agar (SDA), they failed to grow. Similarly, no growth was obtained on RPMI medium with $2 \%$ glucose and Mueller-Hinton agar (MHA). Surprisingly, the isolates grew well on blood agar and chocolate agar. Addition of oxbile (2\%), cholesterol $(20 \mu \mathrm{g} / \mathrm{ml})$ or sheep blood (5\%) to SDA, MHA or RPMI supported good growth of the isolates.

\section{Molecular characterization of the isolates}

Further species-specific identification of the cultured isolates was carried out by PCR sequencing of the internally transcribed spacer (ITS) 1 and ITS2 regions of rDNA and the D1/D2 region sequences within 26S rRNA gene. Genomic DNA from the isolates was prepared and the ITS region of rDNA and D1/D2 regions of 26S rRNA gene were amplified and sequenced as described previously [6,7].

\section{PCR Sequencing of ERG11 and ERG3 genes}

The complete ERG11 gene (including 5' and 3' untranslated regions) was amplified by PCR using $r$ Tth DNA polymerase (Applied Biosystems, Foster City, CA, USA), ERG11F (5' -TCCACCTCGAACCCGTATA-3') and ERG11R (5' -TCCATGTTGATATTCACGATGACT-3') primers and by following the amplification and cycling conditions described previously [8]. The $1923 \mathrm{bp}$ amplicons were sequenced by using Big-Dye terminator cycle sequencing kits (Applied Biosystems) and automated DNA sequencer 3130xl using ERG11FS1 (5'-GAACCCGTATACTCATC TCGTA-3'), ERG11FS2 (5'-GGTGATATCTTCTCTTTC ATGCTA-3'), ERG11FS3 (5'-GACGTGAGAAGAACGA TATCCA-3'), ERG11FS4 (5' -GTTACACTCACTTGCAA GAAGAA-3'), ERG11RS1 (5'-CACGATGACTTACTAT TAGGCTAA-3'), ERG11RS2 (5'-CGAAACCGTAATCA ACTTCGTCA-3'), ERG11RS3 (5'-ATCAAGACACCAA TCAATAGGTT-3'), or ERG11RS4 (5'-AGTAAGCAGCT TCAGCGGAAACA-3') as sequencing primer [8]. The complete ERG3 gene (including 5' and 3' untranslated regions) was also amplified by PCR using rTth DNA polymerase, ERG3F (5'-AGAGATGAGGCCTGGAAG AAGA-3') and ERG3R (5'-AAATATGAGAACCCAGG TCAGCA-3') primers and by following the amplification and cycling conditions described previously [8]. The 1647 bp amplicons were sequenced by using ERG3FS1
(5'-GAAGAAGAGCTGATCTCTCTAGA-3'), ERG3FS2 (5' -TGTCTCTGAATAAGATCGTCTCT-3'), ERG3FS3 (5' -GTACGCCACTTTCATCTTCTTCA-3'), ERG3FS4 (5' -CCTGTTCGACCCTAAGCTAAAGA-3'), ERG3RS1 (5'-CAGGTCAGCACTTGAGTTTTCTCT-3'), ERG3RS2 (5' -GTCTTCTTCTTGTCGGTGTTGT-3'), ERG3RS3 (5'-CTTGTGCAAGGCCTTGTAGACA-3'), or ERG3RS4 (5'-CCTTGTTCACACGCTTCAAGGA-3') as sequencing primer [8]. The complete ERG11 and ERG3 gene sequences were assembled and were compared with the corresponding sequences from reference C. glabrata strain 2001-L5 by using the program Clustal Omega (http://www.ebi.ac.uk/Tools/msa/clustalo/) [5].

\section{Antifungal susceptibility}

Since both bloodstream C. glabrata strains failed to grow on RPMI medium, minimum inhibitory concentrations (MICs) were determined by Etest on RPMI medium containing $2 \%$ glucose and supplemented with cholesterol (20 $\mathrm{\mu g} / \mathrm{ml})$ and Mueller-Hinton agar (MHA) medium supplemented with cholesterol $(20 \mu \mathrm{g} / \mathrm{ml})$ or $5 \%$ sheep blood. For comparison, C. glabrata strain (ATCC 90030) and a recent blood culture isolate (Kw2273/13) were used as controls. The Etest method was performed using Etest strips (bioMérieux, Marcy-ĺEtoile, France) for fluconazole, itraconazole, voriconazole, caspofungin and amphotericin B. A standardized inoculum suspension of each isolate equivalent to a 0.5 McFarland standard was prepared. Plates were inoculated uniformly with cotton swab and allowed to dry before Etest strips were applied. MICs were determined after $48 \mathrm{~h}$ of incubation at $35^{\circ} \mathrm{C}$. Azole MICs were read as the lowest concentrations producing an $80 \%$ reduction of growth. The isolates were considered resistant following the breakpoints described recently for Candida spp. [9].

Since these investigations were part of the routine diagnostic service offered by Mycology Reference Laboratory, no patient consent was required.

\section{Results \\ Growth requirement}

The isolates showed moderate to good growth on blood agar, chocolate agar and routine culture media supplemented with oxbile $(2 \%)$, cholesterol $(20 \mu \mathrm{g} / \mathrm{ml})$ or sheep blood (5\%).

\section{Molecular characterization}

The complete ITS and D1/D2 region sequences of isolates Kw1018/12 and Kw1154/12 were assembled and the corresponding sequences from the two isolates were identical. In BLAST searches (http://www.ncbi.nlm.nih.gov/BLAST/ Blast.cgi?), the ITS and D1/D2 region sequences of isolates Kw1018/12 and Kw1154/12 (EMBL accession nos. HE 993756/7 and HE 998775/6, respectively) showed only 7 
and 1 nucleotide differences with the corresponding sequences from reference C. glabrata strain (CBS 138). The ITS region sequence is also available from another reference C. glabrata strain (ATCC 90030) and showed only 1 nucleotide difference with the corresponding sequences from isolates Kw1018/12 and Kw1154/12. Thus, the ITS and/or D1/D2 region sequences of both the isolates exhibited $>99 \%$ sequence identity with the corresponding sequences from the reference $C$. glabrata strains confirming their identity as C. glabrata. The species-specific identity of the isolates was also confirmed by matrix-assisted laser desorption and ionization-time-of-flight mass spectrometry (MALDI-TOF MS; bioMeriuex) with 99.9\% confidence value.

The ERG11 gene sequences of our isolates (EMBL accession number HF952117) showed four synonymous mutations within the coding region compared to the sequence from reference C. glabrata strain 2001-L5 [5]. Similarly, the ERG3 gene sequences of our isolates (EMBL accession number HF952118) showed three synonymous mutations within the coding region compared to the sequence from reference C. glabrata strain 2001-L5. No non-synonymous mutations were detected and no promoter mutations were apparent in both ERG11 and ERG3 genes.

\section{Antifungal susceptibility}

Both our auxotrophic C. glabrata strains were resistant to amphotericin B (MIC $\geq 32 \mu \mathrm{g} / \mathrm{ml})$, fluconazole (MIC $\geq 256 \mu \mathrm{g} / \mathrm{ml}$ ), voriconazole (MIC $\geq 32 \mu \mathrm{g} / \mathrm{ml}$ ), itraconazole ( $\mathrm{MIC} \geq 32 \mu \mathrm{g} / \mathrm{ml}$ ), and posaconazole ( $\mathrm{MIC} \geq 32 \mu \mathrm{g} / \mathrm{ml}$ ), but appeared susceptible to caspofungin (MIC range 0.064 to $0.19 \mu \mathrm{g} / \mathrm{ml}$ ) on all the media used (Figure 1, Table 1) [9]. In contrast, the MICs of control strain of C. glabrata (ATCC 90030) as well as of a recent blood culture isolate (Kw2273/13) were within susceptible range (Table 1).

\section{Discussion}

A literature review revealed that some strains of C. glabrata require exogenous supply of cholesterol for their growth [10-12]. Both our blood culture isolates grew on blood agar and chocolate agar, but exhibited growth on SDA, RPMI agar or MHA only when supplemented with oxbile (2\%), cholesterol $(20 \mu \mathrm{g} / \mathrm{ml})$ or sheep blood (5\%). Isolation of cholesterol-requiring C. glabrata strains from candidemia patients may not be difficult since patient's blood can serve as a source of cholesterol. However, C. glabrata strains requiring exogenous cholesterol for growth have also been recovered from non-blood specimens and were found to be resistant to antifungal drugs that act on ergosterol biosynthesis [11,12]. Thus, patients infected with such strains may pose significant diagnostic and therapeutic challenges because of lack of growth on routine culture media, but will exhibit in vivo growth due to their ability to maintain integrity of cell

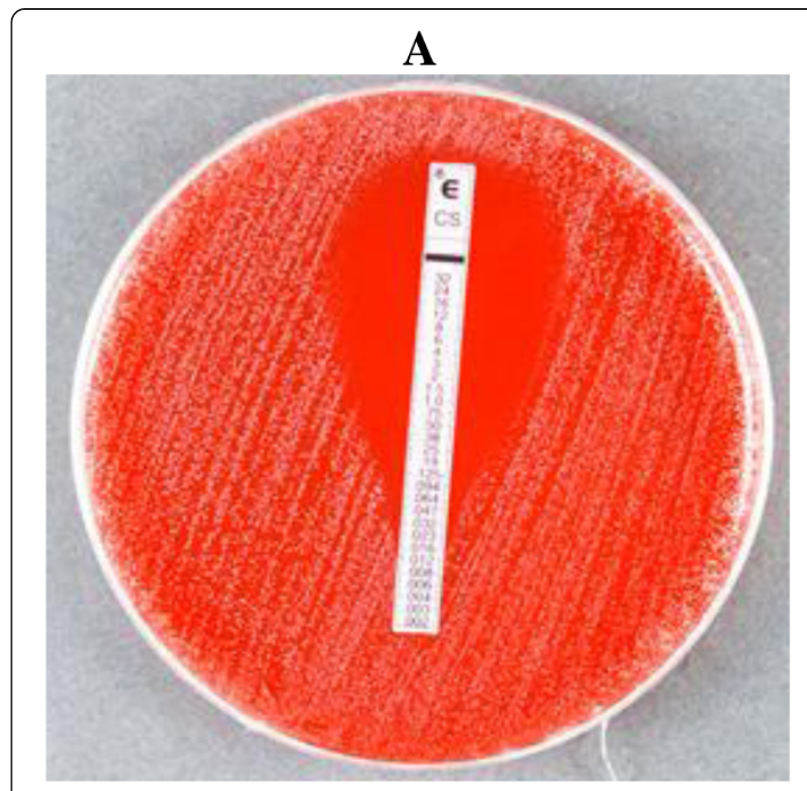

B

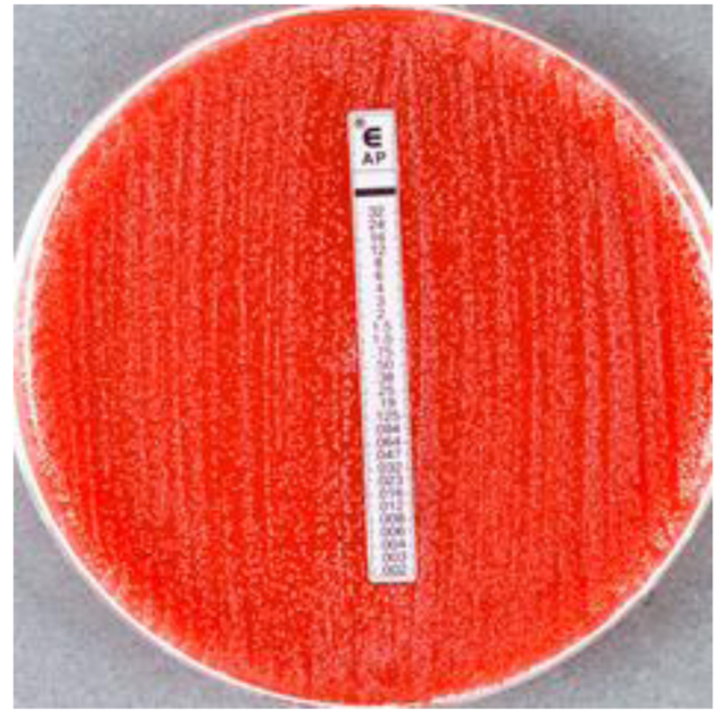

Figure 1 Candida glabrata strain (Kw1018/12) showing Etest MICs for caspofungin (CS, $0.094 \mu \mathrm{g} / \mathrm{ml})(A)$ and amphotericin B $(A P,>32 \mu \mathrm{g} / \mathrm{ml})(B)$ on Mueller-Hinton agar supplemented with $5 \%$ sheep blood and read after $48 \mathrm{~h}$ of incubation at $35^{\circ} \mathrm{C}$.

membrane by utilizing exogenous cholesterol available in blood/tissue milieu. Consequently, such strains can persist at the site of infection in spite of adequate therapy with azoles or amphotericin B. Although cholesteroldependent strains of C. glabrata are rarely encountered, it is possible that isolation of some such strains might be missed if specimens are cultured only on routine media (devoid of a cholesterol source). Here, it is pertinent to mention that C. glabrata is prone to developing reduced susceptibility/cross-resistance to multiple antifungal agents without cholesterol/sterol auxotrophy [13-15]. 
Table 1 Comparative antifungal susceptibility of $C$. glabrata strains on different media with and without cholesterol auxotrophy

\begin{tabular}{|c|c|c|c|c|c|c|c|c|c|c|c|c|c|c|c|c|c|c|c|c|c|c|c|c|}
\hline \multirow[t]{3}{*}{ Strain No } & \multicolumn{24}{|c|}{ Minimum inhibitory concentration $(\mu \mathrm{g} / \mathrm{ml})$ on } \\
\hline & \multicolumn{6}{|c|}{ RPMI with $2 \%$ glucose } & \multicolumn{6}{|c|}{ RPMI with cholesterol $(20 \mu \mathrm{g} / \mathrm{ml})$} & \multicolumn{6}{|c|}{ MHA with cholesterol $(20 \mu \mathrm{g} / \mathrm{ml})$} & \multicolumn{6}{|c|}{ MHA with $5 \%$ sheep blood } \\
\hline & AP & $\mathrm{FL}$ & Vo & IT & PO & CS & AP & FL & vo & IT & POS & CS & AP & FL & vo & IT & POS & CS & AP & $\mathrm{FL}$ & vo & IT & POS & CS \\
\hline Kw1018/12 & - & - & - & - & - & - & $\geq 32$ & $\geq 256$ & $\geq 32$ & $\geq 32$ & $\geq 32$ & 0.125 & $\geq 32$ & $\geq 256$ & $\geq 32$ & $\geq 32$ & $\geq 32$ & 0.19 & $\geq 32$ & $\geq 256$ & $\geq 32$ & $\geq 32$ & $\geq 32$ & 0.094 \\
\hline Kw1154/12 & - & - & - & - & - & - & $\geq 32$ & $\geq 256$ & $\geq 32$ & $\geq 32$ & $\geq 32$ & 0.125 & $\geq 32$ & $\geq 256$ & $\geq 32$ & $\geq 32$ & $\geq 32$ & 0.19 & $\geq 32$ & $\geq 256$ & $\geq 32$ & $\geq 32$ & $\geq 32$ & 0.064 \\
\hline Kw2273/13 & 0.38 & 8 & 0.25 & 6 & 2 & 0.094 & 1 & 4 & 0.125 & 4 & 2 & 0.125 & 1 & 3 & 0.19 & 6 & 0.75 & 0.25 & 0.38 & 3 & 0.19 & 1 & 0.5 & 0.094 \\
\hline ATCC 90030 & 0.19 & 6 & 0.5 & 8 & 4 & 0.094 & 0.5 & 3 & 0.064 & 6 & 4 & 0.125 & 0.38 & 6 & 0.25 & 3 & 6 & 0.19 & 0.38 & 3 & 0.094 & 0.5 & 0.25 & 0.094 \\
\hline
\end{tabular}


The development of cholesterol auxotrophy seemingly does not affect susceptibility to caspofungin or other echinocandins, which is the treatment of choice for $C$. glabrata infections [16].

The patient from whom the two isolates (Kw1018/12 and Kw1154/12) were recovered was initially treated with liposomal amphotericin B (AmBisome, $5 \mathrm{mg} / \mathrm{kg}$ ) for three weeks and later with caspofungin (70 mg loading dose, followed with $50 \mathrm{mg}$ daily dose) for 7 days. However, the patient died of multi-organ failure and septic shock. It is probable that the appropriate therapy with caspofungin was delayed in our patient until C. glabrata was isolated and its resistance against amphotericin $\mathrm{B}$ and azoles was determined.

The major mechanisms known to mediate resistance to azoles in C. glabrata involve upregulation and/or other mutations in ERG11 and efflux pumps. However, resistance to azoles in our isolates was not due to mutations in ERG11 gene and non-synonymous mutations were also not detected in the ERG3 gene. Although the nucleotide sequences of both genes varied slightly, no promoter mutations were detected and the encoded protein sequences (EMBL accession numbers HF952117 and HF952118) were also identical to reference C. glabrata strain 2001-L5 [5]. Upregulation of efflux pumps was not studied in the present investigation. Thus, the molecular basis of resistance to azoles in our isolates remained unidentified. It is probable that upregulation of efflux pumps or mutation(s) in some other genes that have been recently shown to confer resistance to antifungal drugs in C. glabrata are involved in our isolates [3,17-19]. Occurrence of such strains poses a major threat for proper management of such patients, particularly when resistance to echinocandins among C. glabrata strains is also emerging rapidly [20].

\section{Conclusion}

Two cholesterol-dependent C. glabrata strains isolated from the blood of a candidemic patient are described. The report demonstrates that induction of cholesterol/ sterol auxotrophy in C. glabrata may impart resistance to antifungal drugs targeting ergosterol biosynthesis. The observations may have therapeutic implications. Early therapy with echinocandins may be optimal to overcome the problem of triazole/amphotericin B resistance in such isolates. A limitation of our study is that the molecular basis of resistance to azoles in our isolates remained unidentified as only two genes involved in ergosterol biosynthesis were studied while efflux pumps that can also contribute towards resistance to azoles were not investigated. To our knowledge, this is the first report on the isolation of cholesterol-dependent strains of C. glabrata from a candidemia patient exhibiting resistance to azoles and amphotericin B.

\section{Abbreviation}

AP: Amphotericin B; FL: Fluconazole; VO: Voriconazole; IT: Itraconazole; POS: Posaconazole; CS: Caspofungin.

\section{Competing interests}

The authors declare that they have no competing interests.

\section{Authors' contributions}

ZUK and SA drafted the manuscript. LJ performed antifungal susceptibility and molecular identification studies. KA collected the clinical data. All authors read and approved the final version of the manuscript.

\section{Acknowledgements}

The authors are thankful to Ms. Sandhya Vayalil and Rachel Chandy for technical support.

\section{Author details}

${ }^{1}$ Department of Microbiology, Faculty of Medicine, Kuwait University, P. O. Box 24923, Safat 13110, Kuwait. ${ }^{2}$ Microbiology Unit, Al-Amiri Hospital, Safat, Kuwait.

Received: 27 October 2013 Accepted: 31 March 2014

Published: 8 April 2014

\section{References}

1. Diekema D, Arbefeville S, Boyken L, Kroeger J, Pfaller M: The changing epidemiology of healthcare-associated candidemia over three decades. Diagn Microbiol Infect Dis 2012, 73:45-48.

2. Pfaller MA: Antifungal drug resistance: mechanisms, epidemiology, and consequences for treatment. Am J Med 2012, 125(Suppl):S3-13.

3. Sanguinetti M, Posteraro B, Fiori B, Ranno S, Torelli R, Fadda G: Mechanisms of azole resistance in clinical isolates of Candida glabrata collected during a hospital survey of antifungal resistance. Antimicrob Agents Chemother 2005, 49:668-679.

4. Hull CM, Parker JE, Bader O, Weig M, Gross U, Warrilow AG, Kelly DE, Kelly SL: Facultative sterol uptake in an ergosterol-deficient clinical isolate of Candida glabrata harboring a missense mutation in ERG11 and exhibiting cross-resistance to azoles and amphotericin B. Antimicrob Agents Chemother 2012, 56:4223-4232.

5. Geber A, Hitchcock CA, Swartz JE, Pullen FS, Marsden KE, Kwon-Chung KJ, Bennett JE: Deletion of the Candida glabrata ERG3 and ERG11 genes: effect on cell viability, cell growth, sterol composition, and antifungal susceptibility. Antimicrob Agents Chemother 1995, 39:2708-2717.

6. Khan ZU, Ahmad S, Hagen F, Fell JW, Kowshik T, Chandy R, Boekhout T: Cryptococcus randhawai sp. nov., a novel anamorphic basidiomycetous yeast isolated from tree trunk hollow of Ficus religiosa (peepal tree) from New Delhi, India. Antonie Van Leeuwenhoek 2010, 97:253-259.

7. Mokaddas E, Khan ZU, Ahmad S: Prevalence of Candida dubliniensis among cancer patients in Kuwait: a 5-year retrospective study. Mycoses 2011, 54:e29-34

8. Asadzadeh M, Ahmad S, Al-Sweih N, Khan ZU: Rapid molecular differentiation and genotypic heterogeneity among Candida parapsilosis and Candida orthopsilosis strains isolated from clinical specimens in Kuwait. $J$ Med Microbiol 2009, 58(Pt 6):745-752.

9. Pfaller MA, Diekema DJ: Progress in antifungal susceptibility testing of Candida spp. by use of Clinical and Laboratory Standards Institute broth microdilution methods, 2010 to 2012. J Clin Microbiol 2012, 50:2846-2856.

10. Bard M, Sturm AM, Pierson CA, Brown S, Rogers KM, Nabinger S, Eckstein J, Barbuch R, Lees ND, Howell SA, Hazen KC: Sterol uptake in Candida glabrata: rescue of sterol auxotrophic strains. Diagn Microbiol Infect Dis 2005, 52:285-293.

11. Hazen KC, Stei J, Darracott C, Breathnach A, May J, Howell SA: Isolation of cholesterol-dependent Candida glabrata from clinical specimens. Diagn Microbiol Infect Dis 2005, 52:35-37.

12. Rezusta A, Aspiroz C, Boekhout T, Cano JF, Theelen B, Guarro J, Rubio MC: Cholesterol-dependent and amphotericin $B$ resistant isolates of a Candida glabrata strain from an Intensive Care Unit patient. Med Mycol 2008, 46:265-268.

13. Khan ZU, Ahmad S, Al-Obaid I, Al-Sweih NA, Joseph L, Farhat D: Emergence of resistance to amphotericin $B$ and triazoles in Candida glabrata vaginal isolates in a case of recurrent vaginitis. J Chemother 2008, 20:488-491. 
14. Krogh-Madsen M, Arendrup MC, Heslet L, Knudsen JD: Amphotericin B and caspofungin resistance in Candida glabrata isolates recovered from a critically ill patient. Clin Infect Dis 2006, 42:938-944.

15. Pfaller MA, Castanheira M, Lockhart SR, Ahlquist AM, Messer SA, Jones RN: Frequency of decreased susceptibility and resistance to echinocandins among fluconazole-resistant bloodstream isolates of Candida glabrata. J Clin Microbiol 2012, 50:1199-1203.

16. Pappas PG, Kauffman CA, Andes D, Benjamin DK Jr, Calandra TF, Edwards JE Jr, Filler SG, Fisher JF, Kullberg BJ, Ostrosky-Zeichner L, Reboli AC, Rex JH, Walsh TJ, Sobel JD: Infectious Diseases Society of America. Clinical practice guidelines for the management of candidiasis: 2009 update by the Infectious Diseases Society of America. Clin Infect Dis 2009, 48:503-535.

17. Vermitsky JP, Earhart KD, Smith WL, Homayouni R, Edlind TD, Rogers PD: Pdr1 regulates multidrug resistance in Candida glabrata: gene disruption and genome-wide expression studies. Mol Microbiol 2006, 61:704-722.

18. Tsai HF, Sammons LR, Zhang X, Suffis SD, Su Q, Myers TG, Marr KA, Bennett JE: Microarray and molecular analyses of the azole resistance mechanism in Candida glabrata oropharyngeal isolates. Antimicrob Agents Chemother 2010, 54:3308-3317.

19. Farahyar S1, Zaini F, Kordbacheh P, Rezaie S, Safara M, Raoofian R, Heidari M: Overexpression of aldo-keto-reductase in azole-resistant clinical isolates of Candida glabrata determined by CDNA-AFLP. Daru 2013, 21:1.

20. Lewis JS 2nd, Wiederhold NP, Wickes BL, Patterson TF, Jorgensen JH: Rapid emergence of echinocandin resistance in Candida glabrata resulting in clinical and microbiologic failure. Antimicrob Agents Chemother 2013, 57:4559-4561.

doi:10.1186/1471-2334-14-188

Cite this article as: Khan et al:: Isolation of cholesterol-dependent, multidrug-resistant Candida glabrata strains from blood cultures of a candidemia patient in Kuwait. BMC Infectious Diseases 2014 14:188.

\section{Submit your next manuscript to BioMed Central and take full advantage of:}

- Convenient online submission

- Thorough peer review

- No space constraints or color figure charges

- Immediate publication on acceptance

- Inclusion in PubMed, CAS, Scopus and Google Scholar

- Research which is freely available for redistribution 